\title{
Cognitive plasticity in normal and pathological aging
}

This article was published in the following Dove Press journal:

Clinical Interventions in Aging

30 December 20II

Number of times this article has been viewed

\section{Rocío Fernández- \\ Ballesteros' \\ Juan Botella' \\ María Dolores Zamarrón' \\ María Ángeles Molina' \\ Emilia Cabras' \\ Rocío Schettini' \\ Lluis Tárraga ${ }^{2}$}

'Autonomous University of Madrid, Madrid, Spain; ${ }^{2}$ ACE Foundation, Catalonian Institute of Applied

Neurosciences, Barcelona, Spain
Correspondence: R Fernández-Ballesteros Dept of Psychobiology and Health, Autonomous University of Madrid, Campus Cantoblanco, 28049 Madrid, Spain

Tel +34 9l 4975 I8I

Fax +34 9l 49752I5

Email r.fballesteros@uam.es
Abstract: The main goal of the present study is to examine to what extent age and cognitive impairment contribute to learning performance (cognitive plasticity, cognitive modifiability, or learning potential). To address this question, participants coming from four studies (Longitudinal Study of Active Aging, age range, 55-75 years, $N=458$; Longitudinal Study in the very old [90+], age range, 90-102, $\mathrm{N}=188$, and Cognitive Plasticity within the Course of Cognitive Impairment, 97 "Normal", 57 mild cognitive impairment [MCI], and 98 Alzheimer's disease [AD] patients) were examined through a measure of verbal learning (developed from Rey). The results show that all age, MCI, and AD groups learned across the five learning trials of that test, but significant differences were found due to age, pathology, and education. The effects of pathology (MCI and AD) can be expressed in a metric of "years of normal decline by age"; specifically, being MCI means suffering an impairment in performance that is equivalent to the decline of a normal individual during 15 years, whereas the impact of $\mathrm{AD}$ is equivalent to 22.7 years. Likewise, the improvement associated with about 5 years of education is equivalent to about 1 year less of normal aging. Also, the two pathological groups significantly differed from "normal" groups in the delayed trial of the test. The most dramatic difference is that between the "normal" group and the AD patients, which shows relatively poorer performance for the $\mathrm{AD}$ group in the delayed trial than in the first learning trial. The potential role of this unique effect for quick detection purposes of $\mathrm{AD}$ is assessed (in the 75-89 years age range, sensitivity and specificity equal 0.813 and 0.917 , respectively).

Keywords: cognitive plasticity, cognitive modifiability, learning age, aging, Alzheimer's disease, mild cognitive impairment

\section{Introduction}

Cognitive aging research shows a clear picture characterized by a gradual decline in function over time, starting early in life. ${ }^{1-4}$ From the last decades of the 20th century, learning potential, ${ }^{5}$ cognitive modifiability, ${ }^{6}$ or cognitive plasticity across the aging process (also called reserve capacity or testing-the-limit) has been a central issue in gerontology since a stereotypic trait in general population said that "older people are unable to learn." " Cognitive plasticity is operationalized as the extent to which an individual can improve his/her performance in a given cognitive task through training (this procedure has also been called "dynamic assessment" as opposed to the standard "static" measures of cognitive functioning). ${ }^{8-12}$ Although cognitive plasticity measures seem to be preserved during normal aging, many authors agree that they are associated with age; in other words, cognitive plasticity measures have shown a profile of decline in most of the studies. ${ }^{13}$ 
In order to examine plasticity (through episodic memory tasks), Singer et a ${ }^{14}$ trained a mnemonic skill to survivors of the Berlin Aging Study ( $\mathrm{N}=96$; mean age, 84 years; range, 75-101 years). Gains after mnemonic training were modest and most individuals were unable to improve their performance after training; the authors concluded that in very old age, "biological factors are a prominent source of individual differences in plasticity."

Taking as a basis these and other results, Baltes and Smith ${ }^{15}$ considered that the oldest-old, called the "fourth age," "entails a level of biocultural incompleteness, vulnerability, and unpredictability that is distinct from the positive views of the third age (young-old)"; the authors concluded that the oldest-olds are at the limits of their functional capacity.

Nevertheless, a somewhat different conclusion was reached by Yang et al ${ }^{16}$ using a self-guided retest paradigm. This paradigm allows the study of a basic form of cognitive plasticity, called retest learning, as reflected in improvements of performance through retest practice in five trials. They investigated whether cognitive plasticity could be extended from the young-old to the oldest-old. The results showed evidence for continued plasticity until age 80 and above; substantial improvements in performance, comprising one standard deviation from the pre-test to the sixth trial, were observed.

However, research on cognitive functioning in individuals aged 65-90 years who have abnormal memory functioning, but do not meet formal criteria for the diagnosis of dementia, supported Petersen's proposal that the syndrome Mild Cognitive Impairment (MCI) seems to be a transition between normal aging and dementia. ${ }^{17}$ As the author claims: "The concept of the boundary between normal aging and early Alzheimer's disease is a focus of a great deal of research in the field of aging and dementia." In an attempt to determine whether MCI occurred in the oldest-old and whether neuropsychometric performance based on clinical diagnosis was working well in this age group, Boeve et $\mathrm{al}^{18}$ examined with neuropsychometric testing a sample of individuals aged 90-100 years $(\mathrm{N}=111)$. Results yielded 56 normal $(50.45 \%)$, $13 \mathrm{MCI}(11.7 \%)$, and 42 with dementia (37.8\%). The authors concluded that it was possible to observe the full cognitive continuum from normal to MCI to dementia in the oldest-old, but it is important to emphasize that they found significant differences only between the MCI and normal groups in delayed recall. Individuals with MCI were more similar to normal than to patients with dementia.

On the other hand, cognitive plasticity is severely impaired in individuals with dementia, as Baltes and her group pointed out, ${ }^{2,19}$ the assessment of cognitive plasticity (or cognitive reserve capacity) could be a tool for early diagnosis of dementia and an expression of cognitive reserve. In the context of the Berlin Aging Study, Lindenberger and Reischies ${ }^{20}$ reported the results obtained through the Enhanced Cued Recall (ECR) test administered among several neuropsychological tests with the purpose of identifying dementia-specific cognitive impairments and learning potential. Six groups were distinguished: three age groups $(\mathrm{N}=162$; mean ages, 74.9, 84.7, and 94.5 years) and three groups with dementia patients (Mild, $\mathrm{N}=32$; Moderate, $\mathrm{N}=30$; Severe, $\mathrm{N}=31$ ). Persons without dementia showed a decreased level of performance in learning as age advanced but no significant differences were found in learning gains. In comparison with persons with dementia they found significant differences, both in performance levels and in learning gains. Within the dementia subsample, individuals with mild dementia differed from those with moderate or severe dementia with respect to both performance level and learning gain; only those patients with mild dementia obtained similar learning gains than the oldest "normal" groups. In sum, the results indicated that age and dementia had dissociable effects on the recall level and learning gains. Mild dementia patients showed a reduction in performance but they were capable of improvement in the post-test. Finally, individuals with moderate and severe dementia not only showed lower levels of initial recall performance, they did not show any gain after training.

During the last years we have studied cognitive plasticity (or learning potential) in healthy, MCI individuals, and mild Alzheimer's disease (AD) patients. In our studies, cognitive plasticity was assessed through test-training-retest strategy administering several cognitive tasks (visuo-spatial memory, audio-verbal memory, executive function, and verbal fluency), among them the Verbal Memory Learning Potential test $^{21}$ (VMLPt, developed based on the "verbal learning test" from Rey). ${ }^{1}$ Cognitive plasticity was assessed in three groups of older individuals: healthy $(\mathrm{N}=100)$, MCI $(\mathrm{N}=50)$, and AD patients $(\mathrm{N}=50)$. In all tasks the three groups, similar in age and education, improved their performance when training was provided in each task, but healthy elders significantly obtained higher pre-test, post-test, and gain scores than the MCI and AD groups did. There was a gradient of modifiability from healthy to MCI and from MCI to $\mathrm{AD}$, but it must be emphasized that mild AD patients benefited from training as well. Finally, the total score of cognitive plasticity correctly classified $89 \%$ of Healthy, MCI individuals, and $\mathrm{AD}$ patients, and it did better than other tests such as 
the Mini Mental State Examination (MMSE) ${ }^{23}$ In sum, two important findings resulted from this study: (1) Although healthy individuals significantly showed better scores in all cognitive plasticity measures, both MCI individuals and mild AD patients were able to learn, that is, they improved their performance after training in those four cognitive tasks (dots memory, verbal memory, executive function, and verbal fluency), ${ }^{21}$ and (2) AD patients improved their performance in cognitive plasticity measures after 6 months of a psychostimulation program. ${ }^{24}$

Most of the studies in cognitive plasticity have been conducted in Western countries, where the level of education is quite high even in the oldest cohort. Nevertheless, in a previous study, Fernández-Ballesteros and $\mathrm{Calero}^{25}$ found significant differences in learning potential (cognitive plasticity) between elders with high and low levels of education. The effect of education in crystalized intelligence is very well known; ${ }^{26}$ on the clinical field, lack of education is considered a risk factor for accelerated memory decline, on the contrary education is considered a protective factor for cognitive impairment and dementia. ${ }^{27,28}$ For example, Schmand et $\mathrm{al}^{29}$ examined the decline across life span in the population density of neocortical synapses which do not reach the level found by AD patients. Also, they examined the broad effect of education in rising neocortical synaptic density; finally, after considering aging population projections, they concluded that it is important to protect cortical synapses through cognitive stimulation across life span. This panorama make education as a compensator and/or a protective factor for cognitive impairment.

In sum, cognitive plasticity seems to be a relatively new construct yielding useful information about normal and impaired mental functioning being influenced by age, level of pathology, and education. After this review, an important question remains: to what extent are age and pathology accounting for cognitive decline and/or impairment? Thus, considering the growing interest in the oldest-old and the relevance and increasing prevalence of cognitive impairment, dementia due to AD through very old age, the present article addresses three main questions: (1) to what extent cognitive plasticity is preserved but decline across different ages, including the very old, and to what extent this decline is mediated by education; (2) to what extent do MCI individuals and mild Alzheimer's disease patients maintain a gradient of modifiability; and (3) to what differential extent age, pathology, and education influence plasticity.

\section{Methods}

\section{Participants}

The total sample came from four different research projects:

\section{"Normal" older adults, 55-89 years old}

From the baseline of the ELEA Project (Longitudinal Study of Active Aging), ${ }^{30}$ in which people were assessed in the year 2006, individuals from 55 to 74 years of age were recruited. The criterion for inclusion in this population study was being in the age range 55-75 years. In order to have a varied sample, convenient subsamples were recruited from four contexts (a representative sample of the Madrid population, rural and urban citizen clubs, and a university program for the elderly).

Participants aged 75-89 years were assessed in 2002 from the Learning Potential Study (LPS). ${ }^{21}$ In this clinical study, the criteria for inclusion of "normal" older sample were: absence of central nervous system pathological conditions and other health problems related to cognitive impairment (alcoholism, drug addiction, or systemic diseases, etc).

Therefore, a total of 601 participants 55-89 years old were included in the present study (mean age, 68.8 years; $\mathrm{SD}=6.6$; range, 55-89; 347 females and 254 males; mean years of education, 8.9 years; $\mathrm{SD}=9.4$ )

\section{"Normal" oldest-old, older than 90 years old}

From the baseline of the "90+" Longitudinal Project, assessed during 2007, the data from 188 participants older than 90 years (mean age, 92.9; $\mathrm{SD}=2.5$; age range, 90-102; years of education, 9.3 years; $\mathrm{SD}=14.5$ ) were re-analyzed (all of them were born before 1917; 121 females and 67 males). The criteria for inclusion for this population study were the following: $\geq 90$ years old, independence in basic activities of daily living (score $>60$ on the Barthel scale), ${ }^{31}$ and having preserved cognitive capacity (score $>56$ in the Informant Questionnaire of Cognitive Impairment on the Elderly or have less than two errors in the Sort Portable Mental Status Questionnaire (SPMSQ) ${ }^{32}$ or less than three if the level of education is low). This is also a convenience sample, recruited from several contexts.

It is important to mention that our "normal" age groups significantly differed $\left(\chi^{2}[24]=56.43, P<0.001\right)$ in their level of education; younger cohorts had higher levels of education than older cohorts. These differences correspond to population differences in those cohorts due to socio-historical changes in the education system in Spain. ${ }^{33}$

Table 1 shows how the "normal" sample is distributed for age groups $(55-64 ; 65-69 ; 70-74 ; 75-89 ; 90+$ years $)$ and gender. 
Table I "Normal" age groups by gender

\begin{tabular}{llll}
\hline Age group (years) & \multicolumn{2}{l}{ Gender } & \\
\cline { 2 - 3 } & Male & Female & Total \\
\hline $55-64$ & 57 & 98 & 155 \\
$65-69$ & 70 & 96 & 166 \\
$70-74$ & 76 & 107 & 183 \\
$75-89$ & 51 & 46 & 97 \\
$90+$ & 67 & 120 & 187 \\
Total & 321 & 467 & 788 \\
\hline
\end{tabular}

"Mild Cognitive Impairment" and Alzheimer's disease patients

From the baseline of the Longitudinal Project "Cognitive Plasticity during the Course of Dementia CPCD" (baseline in 2007) data from 57 individuals diagnosed as "Mild Cognitive Impairment" (MCI; mean age, 76.11 years; $\mathrm{SD}=5.20$; range, 65-86 years) and 98 patients (mean age, 78.16; $\mathrm{SD}=5.07$; range, 64-88) diagnosed with Alzheimer's disease ("mild" AD) were re-analyzed. Table 2 shows the sample distribution by gender and age. Regarding education, no significant differences between groups were found; the mean years of education were 10.46 years for the MCI group $(\mathrm{SD}=12.7)$ and 7.16 years for the $\mathrm{AD}$ group $(\mathrm{SD}=5.32)$.

$\mathrm{MCI}$ individuals were diagnosed in the Diagnostic Unit of the ACE Centre by independent experts according to the criteria from Petersen et al: ${ }^{34}$ memory complaints, normal activities of daily living, normal general cognitive function, abnormal memory for age, and do not follow dementia criteria.

AD patients were included according to the criteria from the Diagnostic and Statistical Manual of Mental Disorders, Fourth Edition (DSM-IV); ${ }^{35}$ criteria from the National Institute of Neurological and Communicative Disorders and Stroke-Alzheimer's Disease and Related Disorders Association (NINCDS-ADRDA; Protocol Attachment HGIV.3); ${ }^{36}$ a Mini Mental State Examination $(\mathrm{MMSE})^{37}$ score $18-26$ or a level 4 score in the Global Deterioration Scale (GDS); ${ }^{38}$ a Hachinski Scale score ${ }^{39} \leq 4$. Informed consent from a close relative was required. Subjects with psychiatric illness or primary neurological

Table 2 Composition of the pathology groups ( $\mathrm{MCl}$ and $\mathrm{AD}$ ) according to gender and age group

\begin{tabular}{|c|c|c|c|c|c|c|}
\hline & \multicolumn{4}{|c|}{ Age group (years) } & \multicolumn{2}{|c|}{ Gender } \\
\hline & $55-64$ & 65-69 & $70-74$ & $75-89$ & Male & Female \\
\hline $\begin{array}{l}\mathrm{MCl} \\
(\mathrm{N}=57)\end{array}$ & 0 & 7 & 14 & 36 & 28 & 29 \\
\hline $\begin{array}{l}A D \\
(N=98)\end{array}$ & 2 & 4 & 17 & 75 & 57 & 41 \\
\hline & 2 & 11 & 31 & 111 & 85 & 70 \\
\hline
\end{tabular}

Abbreviations: $\mathrm{MCl}$, mild cognitive impairment; $\mathrm{AD}$, Alzheimer's disease. disorder or delirium, or with alcoholism or drug addiction history were excluded.

\section{Instruments and procedures}

In order to assess cognitive plasticity, all participants were evaluated under standard conditions by the Verbal Memory Learning Potential test VMLt (based on the "verbal learning test" from Rey; ${ }^{22,40}$ modified by Fernández-Ballesteros et al). ${ }^{21}$ The task consisted of the auditory presentation of 15 common words which were to be immediately recalled (free recall). The number of words correctly recalled in the first trial was considered the pre-test score or baseline. Afterwards, five consecutive learning trials were performed using the same words; after the second, third, and fourth trials, feedback (number of words correctly recalled) and verbal reinforcement were provided ("good!; you did very well!"); in the fifth trial, a cognitive strategy (verbally described: "perhaps you can group the words") was suggested. Trials 2-5 were considered the training phase. The sixth trial was considered the post-test. Finally, after the presentation of an interference task, delayed recall was included (seventh trial). The number of words correctly recalled in each trial was considered the raw score.

In all the studies, VMLt instructions were the same being administered for a trained psychologist expert on aging. In the two population studies (ELEA and 90+), the VMLt was placed inside an in-home interview, interference phenomena was a four-item Wellbeing Scale; in the two clinical studies (LPS and CPCD), the VMLt was placed into a Cognitive Battery, interference phenomena was a dots task.

\section{Statistical analysis}

In order to examine group differences (age group and pathology) among scores through trials, $t$-tests and between and/or repeated measures analysis of variances (ANOVAs) were conducted with post-hoc Bonferroni comparisons, when appropriate. ANOVAs were performed in order to test the effects of the level of education with the age groups. Finally, the relative weights of age and pathology group performance were assessed via logistic regression. Statistical analyses were carried out using SPSS software (v 15.0; SPSS, Inc, Chicago, IL).

\section{Results}

We first analyzed the learning performance of "normal" participants, and then we compared their performance to that of the pathological groups. 


\section{Performance of the "normal" group}

Figure 1A shows performance levels of the "normal" participants, according to their age group. A $5 \times 7$ ANOVA (five between-group age levels, seven within-subject trials) was applied to the values (it could be argued that trial 7 is qualitatively different from the other six, and should not be included in this analysis; when repeated with only the first six trials, the pattern of results did not change in this group nor in the equivalent analyses for the other groups, in the sense that the same factors remained significant). There was a significant main effect of the age group $(F[4,715]=127.0 ; P<0.001)$ and the trial $(F[6,4290]=812.6 ; P<0.001)$. As revealed in the figure, performance was inversely related to age, and performance monotonically increased along the first six trials but decreased in the delayed trial (seventh trial). The interaction was also statistically significant $(F[24,4290]=15.3$; $P<0.001$ ); again, the functions in Figure 1A show that the rate of performance increase as a function of the trial number (until trial 6) was slower as age increased. When years of education was included as a covariate it explained a significant part of the variance $(F[1,669]=35.5 ; P<0.001)$, and the interaction of trial number by years of education was also significant $(F[6,4014]=2.9 ; P=0.01)$. However, the effects highlighted in the analysis above (age group, trial number, and the age group by trial interaction) remained significant when years of education was included as a covariate. Of course, years of education was positively associated with performance; performance was higher with increasing years of education.

Regarding trial 7, the delayed recall trial, Figure 1A shows that all age groups demonstrated a decline. Although a oneway ANOVA of the five age groups on the differences (T6-T7) reached statistical significance $(F[4,768]=2.4 ; P=0.048)$ there was no systematic pattern in the differences associated with age group. Bonferroni post-hoc comparisons showed that only one pair (55-64 versus 70-74 age groups) from ten possible comparisons differed significantly $(P=0.032)$. The result of this ANOVA is interpreted as a probable type I error, and we conclude that there is no systematic association between age and the amount of decline of performance in the delayed trial.

The upper panel of Table 3 shows some specific comparisons of special interest to our purpose here: gain across the six trials (T6-T1), and decrease in the delayed trial as compared with the level reached in the sixth trial (T6-T7). Performance level at the first trial (T1) was compared across the groups by means of an ANOVA that showed a significant effect $(F[4,779]=65.8 ; P<0.001)$

Figure 1A shows that, as expected, performance decreased monotonically as a function of age. Bonferroni post-hoc comparisons show significant differences between all pairs of age groups $(P<0.05)$ with two exceptions: 65-69 versus 70-74 and 70-74 versus 75-89.

\section{$\mathrm{MCl}$ group}

Figure 1B shows the performance levels of the MCI group according to their ages. A $2 \times 2 \times 7$ ANOVA (two pathology between-groups - normal versus MCI, two between-group age levels, seven within-subject trials) was applied to the values (only the two age groups of 70-74 and 75-89 were included, given the small size of the 65-69 age group). There was a significant main effect of the pathology group $(F[1,365]=54.5 ; P<0.001)$, the age $\operatorname{group}(F(1,365)=4.8$; $P<0.03)$, and the trial number $(F[6,2190]=275.1$; $P<0.001)$; as revealed in the figure performance was lower for the MCI group than for normal participants, performance monotonically increased along trials $1-6$ and decreased in the delayed (seventh) trial, and performance was worse for the older group. The first-order interaction between the pathology group and the trial was significant $(F[6,2190]=37.3$;
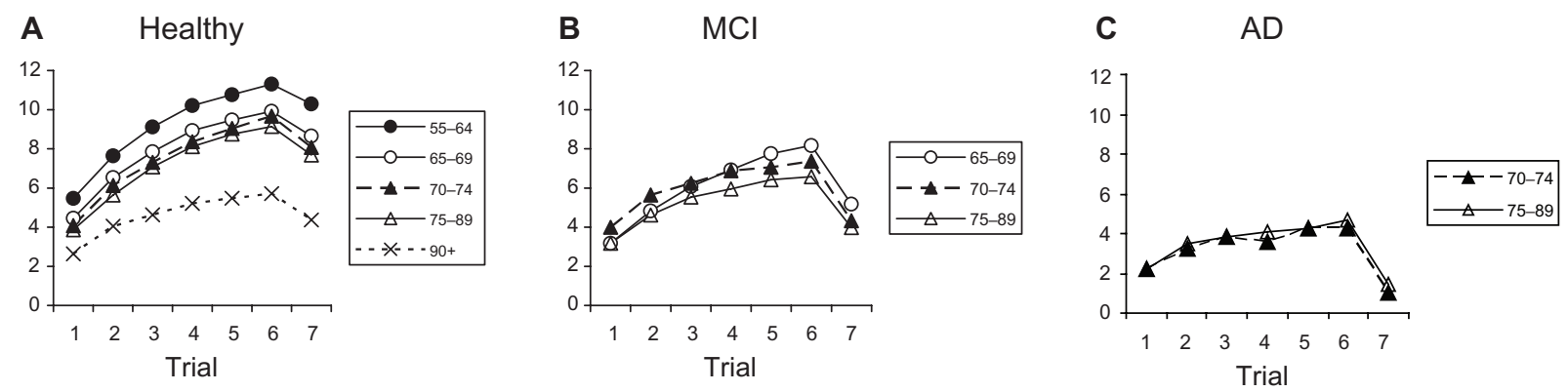

Figure I Learning performance on (A) healthy elders, $(\mathbf{B}) \mathrm{MCl}$ individuals, and $(\mathbf{C}) \mathrm{AD}$ patients. The mean number of words correctly recalled ( $y$-axis) is plotted as a function of the trial ( $1-7$ in the $\mathrm{x}$-axis), for each age group. The first trial is considered as the baseline, whereas trials $2-5$ are the training phase, and trial 6 is considered the post-test; the 7th trial is a delayed trial performed (see the text).

Abbreviations: $\mathrm{MCl}$, mild cognitive impairment; $\mathrm{AD}$, Alzheimer's disease. 
Table 3 Learning comparison in gain score (Trial 6-Trial I) and delayed scores (Trial 6-Trial 7), $t$-test

\begin{tabular}{|c|c|c|c|c|}
\hline Group & $\begin{array}{l}\text { Age group } \\
\text { (years) }\end{array}$ & Comparison & Mean diff & \\
\hline \multirow[t]{10}{*}{ Normal } & $55-64$ & T6-TI & 5.85 & $\begin{array}{l}t(154)=29.85 \\
P<0.001\end{array}$ \\
\hline & & $\mathrm{T} 6-\mathrm{T7}$ & 1.02 & $\begin{array}{l}t(154)=6.82 \\
P<0.001\end{array}$ \\
\hline & $65-69$ & T6-TI & 5.46 & $\begin{array}{l}t(165)=32.48 \\
P<0.001\end{array}$ \\
\hline & & T6-T7 & 1.26 & $\begin{array}{l}t(165)=8.61 \\
P<0.001\end{array}$ \\
\hline & $70-74$ & T6-TI & 5.59 & $\begin{array}{l}t(181)=29.70 \\
P<0.001\end{array}$ \\
\hline & & $\mathrm{T} 6-\mathrm{T7}$ & 1.63 & $\begin{array}{l}t(180)=12.19 \\
P<0.001\end{array}$ \\
\hline & $75-89$ & T6-TI & 5.28 & $\begin{array}{l}t(96)=22.39 \\
P<0.001\end{array}$ \\
\hline & & $\mathrm{T} 6-\mathrm{T7}$ & 1.51 & $\begin{array}{l}t(95)=8.09 \\
P<0.001\end{array}$ \\
\hline & $\geq 90$ & T6-TI & 3.08 & $\begin{array}{l}t(172)=\mid 8.0 I ; \\
P<0.00 I\end{array}$ \\
\hline & & $\mathrm{T} 6-\mathrm{T7}$ & 1.29 & $\begin{array}{l}t(170)=7.83 \\
P<0.00 I\end{array}$ \\
\hline \multirow[t]{4}{*}{$\mathrm{MCl}$} & $70-74$ & T6-TI & 3.36 & $\begin{array}{l}t(32)=18.01 \\
P<0.001\end{array}$ \\
\hline & & T6-T7 & 3.03 & $\begin{array}{l}t(32)=7.83 \\
P<0.001\end{array}$ \\
\hline & $75-89$ & T6-TI & 3.40 & $\begin{array}{l}\mathrm{t}(59)=8.78 \\
P<0.00 \mathrm{I}\end{array}$ \\
\hline & & T6-T7 & 2.61 & $\begin{array}{l}t(58)=10.07 \\
P<0.001\end{array}$ \\
\hline \multirow[t]{4}{*}{$A D$} & $70-74$ & T6-TI & 2.06 & $\begin{array}{l}t(16)=4.35 \\
P<0.001\end{array}$ \\
\hline & & T6-T7 & 3.24 & $\begin{array}{l}t(16)=7.78 \\
P<0.00 I\end{array}$ \\
\hline & $75-89$ & T6-TI & 2.48 & $\begin{array}{l}t(74)=11.40 \\
P<0.001\end{array}$ \\
\hline & & $\mathrm{T} 6-\mathrm{T7}$ & 3.28 & $\begin{array}{l}t(74)=14.42 \\
P<0.001\end{array}$ \\
\hline
\end{tabular}

Abbreviations: $\mathrm{MCl}$, mild cognitive impairment; $\mathrm{AD}$, Alzheimer's disease.

$P<0.001$ ); the functions in Figure 1A and B show that the rate of performance increase as a function of trial was slower for the MCI group. However, the first-order interactions of age group were not significant, neither with trial number $(F[6,2190\})=0.524 ; P=0.79)$, or the pathology group $(F[1,365]=0.675 ; P=0.412)$; in the same vein, the secondorder interaction (pathology group by age group by trial) was not significant $(F[6,2190]=0.437 ; P=0.854)$.

In the previous section a significant interaction between age and trial was found. However, Figure 1A suggests that it could be due to the extreme age groups (55-64 and 90+) that were not present in the MCI group. Repeating the ANOVA of the previous section for the normal participants, with only the two age groups with enough data in the MCI group (70-74 and 75-89), it was found that the interaction between trial and age group was not significant $(F(6,1374)=1.4 ; P=0.214)$. That is, the interaction of the previous analysis was due to the extended range of age groups.

When adding years of education as a covariate, a significant increase in the variance was explained $(F[1,219]=6.6$; $P=0.011$ ), and the interaction with trial was not significant $(F[6,1314]=0.79 ; P=0.579)$. However, the effects highlighted (pathology group, age group, trial, and pathology by trial interaction) remained significant when the covariate was years of education.

For the decline of performance as a dependent variable (difference between performance in the last learning trial and the delayed trial, T6-T7), a $2 \times 2$ ANOVA (two pathology groups, two age groups) was performed. The results showed no significant effect of the main factor age group $(F[1,359]=1.27 ; P=0.261)$, nor of the interaction $(F[1,359]=1.58 ; P=0.209)$, but a significant effect of the pathology group $(F[1,359]=28.0 ; P<0.001)$. The decline was significantly greater for the MCI group than for the normal subjects (2.81 versus 1.62, on average).

The middle panel of Table 3 shows the specific comparisons for the MCI age groups for which there were enough participants. Again, the two age groups showed significant gains and delayed decrease. Furthermore, alongside the first trial performance, a $2 \times 2$ ANOVA (two pathology groups, two age groups) was performed. It showed nonsignificant effects of pathology group $(F[1,369]=1.62 ; P=0.424)$, the group $(F[1,369]=2.96 ; P=0.335)$, and the interaction $(F[1,369]=2.69 ; P=0.102)$.

In short, the results showed that when taking normal subjects of a wide age range, level of performance changes significantly as a function of age. The MCI group showed a level of performance comparable to that of the normal subjects in the first trial. However, the learning curves show slower rates of improvement for the MCI subjects, and a larger decrease in the delayed trial. Although the slope of the learning curve across age was smaller for the MCI group, this interaction was not significant.

\section{AD group}

Figure $1 \mathrm{C}$ shows the performance levels of the AD group, according to their age group; data were only available for subjects from the 70-74 and 75-89 age groups. Some analyses comparing the two pathological groups were run. A $2 \times 2 \times 7$ ANOVA (two pathology between-group, two between-group age levels, seven within-subject trials) was 
applied to the values. There was a significant main effect of the pathology group $(F[1,220]=70.5 ; P<0.001)$, and trial $(F[6,1320]=110.3 ; P<0.001)$, but not of the age group $(F[1,220]=0.254 ; P=0.615)$. As revealed in the figure, performance was lower for the $\mathrm{AD}$ group than for the $\mathrm{MCI}$ participants, and performance monotonically increased along trials 1-6 and decreased in the delayed (seventh) trial. The first-order interaction between pathology group and trial was significant $(F[6,1320]=6.62 ; P<0.001)$; the functions in Figure 1 show that the rate of performance increase as a function of the trial was slower for the AD group. However, the first-order interactions of the age group were not significant, nor was the trial $(F[6,1320]=0.658 ; P=0.684)$, or the pathology group $(F[1,220]=3.487 ; P=0.063)$; in the same vein, the second-order interaction (pathology group by age group by trial) was not significant $(F[6,1320]=0.628 ; P=0.708)$.

When adding the years of education as a covariate, it did not explain a significant part of the variance $(F[1,137]=0.018 ; P=0.894)$, and the interaction with trial was not significant $(F[6,822]=1.683 ; P=0.122)$. However, the effects highlighted (pathology group, trial, and the pathology group by trial interaction) remained significant when the covariate was years of education.

Again taking decline of performance as a dependent variable, a $2 \times 2$ ANOVA (two pathology groups, two age groups) was performed. The results show no significant effect of the main factors age group $(F[1,220]=0.318 ; P=0.573)$, pathology group $(F[1,220]=1.617 ; P=0.205)$, nor of the interaction $(F[1,220]=1.264 ; P=0.262)$. The decline was statistically equivalent for the two pathology groups and age levels.

The lower panel of Table 3 shows the specific comparisons for the age groups of AD for which we had enough participants. Again, the two age groups showed significant gain and delayed decrease. Furthermore, we ran with the first trial performance a $2 \times 2$ ANOVA (two pathology groups, two age groups). It did not show significant effects for either age group $(F[1,181]=2.60 ; P=0.109)$ or the interaction $(F[1,181]=2.66 ; P=0.105)$, but showed a significant effect of the pathology group $(F[1,181]=28.89 ; P<0.001)$; as expected, the direction of the effect was that $\mathrm{AD}$ participants showed a lower performance than MCI participants (2.24 vs 3.59 , respectively).

\section{The Verbal Memory Learning Potential test as a diagnostic tool}

The average values from Figure 1 suggest that the learning test employed could be a useful tool for quick classification or screening to discriminate between healthy individuals and $\mathrm{AD}$ patients. Especially interesting is the fact that the only group that showed lower performance in the delayed trial (trial 7) than in the first learning trial (trial 1) was the $\mathrm{AD}$ individuals. After checking other combinations, the best tool was found to be the difference between the levels of performance in the seventh and first trials (T7-T1). If individuals are categorized as $\mathrm{AD}$ with the criterion of having a difference of 0 or negative $((\mathrm{T} 7-\mathrm{T} 1) \leq 0)$, the sensitivity for the 70-74 age group equals 0.824 (14/17), whereas the specificity equals $0.918(168 / 183)$. These statistics are about the same in the $75-89$ age group, as sensitivity is 0.813 (61/75) and specificity is 0.917 (88/96). However, there were insufficient participants for this same calculation to be done in other age groups.

In short, the difference between performance in the delayed (seventh) trial and the first trial can be used as a simple diagnostic tool, at least in the interval of ages between 70 and 89 years. Classifying as AD those who do not show better performance in trial 7 compared with their performance in trial $1(\mathrm{~T} 7-\mathrm{T} 1 \leq 0)$ yields a good balance between sensitivity and specificity.

\section{Assessing the impact of disease in terms of normal aging}

This section considers how the association of impairment with pathology can be expressed in units that can be more easily understood. Specifically, a comparison between Figure $1 \mathrm{~A}-\mathrm{C}$, allows us to ask the following question: can the performance of the $\mathrm{MCI}$ and $\mathrm{AD}$ groups be placed somewhere on a continuum of decline in normal individuals? Or, in other words, could the impairment associated with the pathology be converted (translated) into years of normal decline? How many years of normal impairment are equivalent to the impairment associated to the pathology, when both are reflected in a single measure?

In order to analyze the impact of pathology (MCI and AD) in terms of the impact of age, we adjusted some regression models. In the final model, performance in the 6th trial was the dependent variable, and age (not grouped) plus the years of education and two dummy variables that code the pathology group, were the independent variables. All independent variables were entered in the equation, as all explained a significant part of the variance. The final model, for which $R^{2}$ (adjusted $)=0.467$, was:

$$
\begin{aligned}
T 6^{\prime}= & 20.52-0.160 \cdot A g e-2.395 \cdot M C I-3.627 \cdot A D \\
& +0.034 \cdot \text { YofEd }
\end{aligned}
$$


Some simple calculations allow estimation of the impact of having MCI in the 6th trial in terms of years of normal decline. Specifically, dividing the slopes $(2.395 / 0.16)$ yield that having MCI means suffering an impairment in performance that is equivalent to the decline of a normal individual during 15 years; the same analysis for $\operatorname{AD}(3.627 / 0.16=22.7)$ yield that the impact of being $\mathrm{AD}$ is equivalent to 22.7 years of normal decline in performance. In other words, the regression model predicts (in trial 6) for an MCI individual the same performance as to a normal individual with 15 years more than them; it also predicts for an $\mathrm{AD}$ individual the same performance as to a normal individual with 22.7 years more than them. Of course, the years of education have a positive impact; expressed also in a "years of normal decline" metric, the impairment that in the average person has associated 1 year of normal aging $(0.16 / 0.034)$ is equivalent to the improvement associated to about 5 years of education.

The same does not happen with the delay trial (T6-T7) or, in other words, with the interference phenomenon. The correlation between age and delay trial performance was not significant $(R=0.038 ; P=0.287)$. That is, whereas age can account for a decreased gain across the six trials and the impact of MCI and $\mathrm{AD}$ can be assessed in terms of normal aging, the decline associated with the delayed trial is not related to normal aging, but both $\mathrm{MCI}$ and $\mathrm{AD}$ have an important impact on it. In other words, the interference phenomenon is not an age-based phenomenon but a sign of pathology.

\section{Discussion}

All age groups of normal individuals show plasticity as reflected in performance increases along the six trials of verbal learning. The effect of age is clear in the first trial, as performance is lower as age increases, and also in the rate of learning, as the interaction between trial and the age group indicates that people learn at a slower rate the older they are. In other words, in terms of plasticity theory ${ }^{1}$ or dynamic assessment, "static" assessment of cognitive performance as well as learning performance ("dynamic" assessment) are both related to age. On the contrary, the drop-in performance from the 6th to the 7th (delayed) trial is not associated with age but to pathology.

In terms of this pathology, the $\mathrm{AD}$ group shows lower performance in the first trial than the MCI and normal individuals of the same age; this is a specific qualifier for $\mathrm{AD}$ persons. The rate of learning for the three groups follows a continuum (normal $>\mathrm{MCI}>\mathrm{AD}$ ). The drop-in performance in the delayed trial is significantly larger for the two pathological groups; there is no significant difference between them. This is a shared qualifier for the pathological groups. Differences between normal and $\mathrm{AD}$ individuals match the Baltes and Raykov $^{2}$ and Lindenberger and Richeis ${ }^{20}$ results as well as our own previous studies regarding normal, MCI individuals, and $\mathrm{AD}$ patients..$^{21,23}$

Regarding our research questions, the first one refers to what extent cognitive plasticity, assessed through verbal learning, declines across age, including the oldest-old. According to our results, all age groups from 55 through older than 90 learn from cognitive training and, therefore, they show cognitive plasticity as reflected in an increased performance along a learning curve of six trials. The effect of age is clear in the first trial, as baseline performance is lower as age increases, but there is an effect of age in the rate of learning as well, as the interaction between the trial and the age group indicates that people learn at a slower rate as age increases. On the contrary, the strong effect of interference showed in the delayed trial (difference of performance between trials 6 and 7) is not associated with age but is a specific characteristic of the impaired groups.

In sum, our findings provide evidence for differential learning performance across aging including the very old, and they converge with other studies with young-old ${ }^{1,13,25}$ and with the oldest-old. ${ }^{16,20}$ Regarding learning decline, it is important to mention that we did not find significant differences between two groups: 70-74 years and 75-89 years; that is, in our data there is no decline of learning from 70 to 89 years, as has been reported by Yang et al. ${ }^{16}$

Regarding our very old nonagenarian participants, their decline in learning is significantly higher than that of the other age groups, but this age group continues to improve their learning performance. This supports the idea that cognitive plasticity as demonstrated in the young-old can be extended into the oldest-old, as has been reported by Lindenberger and Reschies..$^{20}$ Nevertheless, their performance in learning is similar to the decline of those younger individuals diagnosed as having MCI; that is, they show no significant differences in their learning curve with MCI individuals, with the exception of the 7th delayed trial. At this point we might conclude, as Baltes and Smith ${ }^{15}$ do, that in spite of the fact that they constitute a group of independent nonagenarians, they perform as cognitive impaired individuals. Could it be considered at the limits of their biological capacity? From our point of view, we cannot reach that conclusion; given that our group of nonagenarians belongs to a cohort for whom mandatory education did not exist (almost half of them have no formal education). Even then, our analysis shows that 
when education is introduced as a covariant, age differences between nonagenarians and the other age groups continue to be significant. It can be stated that our nonagenarian sample is at the limits of their under-stimulated capacities. Education seems to be a trigger for learning and we must be aware that our very old participants had a very low education level, which is different from the younger cohorts.

As Terry and Katzman ${ }^{41}$ proposed, policies have to take into consideration the importance of education across lifespan. Moreover, the importance of life-long learning seems to be a new platform for improving plasticity along life and in old age. But it is also important to fight against social and group stereotypes which propagate the idea that older persons cannot learn or are unable to solve problems.

Also, it is important to investigate to what extent cognitive training across the lifespan and during old age can play a role in learning capacities, as pointed out by Mayr. ${ }^{10}$ Additionally, in our data coming from the study of individuals of $90+$ years, those nonagenarians who are cognitively active (that is, in the baseline performed cognitive activities such as "reading", "playing chess") show better cognitive functioning in the follow-up, after 6-14 months. ${ }^{42}$ In other words, a new panorama is open for the aging mind.

Regarding our second goal, that is, to what extent MCI individuals and mild AD patients maintain a certain level of plasticity or gradient of modifiability, as in previous studies, both cognitive-impaired groups show genuine learning. Although there are significant differences in learning between "normal" and "impaired" groups, MCI and AD individuals can modify their performance through cognitive training. As expected, the baseline score was significantly lower in the AD than in MCI groups. The rate of learning is different for the three groups (normal $>\mathrm{MCI}>\mathrm{AD}$ ). But the decline of performance in the delayed trial is significantly larger for the two pathological groups. Moreover, there is no difference between them; this is a shared qualifier for the pathological groups. Finally, not showing higher performance in the delayed trial than in the first trial can be used as a quick diagnostic or screening tool for AD. ${ }^{21,23}$

Regarding cognitive plasticity shown by MCI and AD groups, it can be concluded that although they show lower performance, both in the pre-test and in the gain scores, than "normal" individuals (with the exception of the similarities between the oldest-old and the MCI group) both groups reach (after the training) twice the number of words recalled than on the first trial: both groups show the ability to learn. These results are in accordance to Schreiber and Schneider, ${ }^{43}$ who indicate that plasticity-oriented information given in a pre-test-training-post-test-design is potentially useful for the purposes of early identification of dementia, and to Lindenberg and Reschies, ${ }^{20}$ who found that mildly demented individuals, with a lower baseline that those "normal" individuals, show similar gains through learning.

Our results regarding the interference phenomenon show that a lower score in trial 7 than in trial 1 (a kind of "de-learning" effect), is a result specific to the AD group. It constitutes a clear and pathognomonic indicator of dementia, even though delay trial performance in the MCI group also showed significant differences with any other group (including the very old). This result is in accordance to the Petersen's group, ${ }^{18}$ who found differences between nonagenarian demented, with MCI and "normal," just in the delay trials, which is an indicator of neuropsychological pathology. Nevertheless, it is important to emphasize that mild AD patients can take benefit from learning, as already indicated. ${ }^{24}$ Any nihilistic position concerning these patients does not have empirical support; with balanced objectives, they can be trained and take benefit from learning. ${ }^{44}$

Finally, taking into consideration that both age and pathology are accounting for cognitive plasticity variance, our third question refers to the relative weights of both independent variables. Since pathology is associated with age, it is difficult to disentangle both factors. Moreover, our MCI and AD samples are both younger (age range 55-90 years) than our total age range of "normal" sample (55-102 years) and have both nonsignificant but higher and homogenous levels of education. Nevertheless, our analyses support the hypothesis that the effect of having MCI or AD have weights equivalent to 15 and 22.7 years of normal aging, respectively. In the same vein, 1 year of reverse normal aging seems to be equivalent to the improvement associated with about 5 years of education.

Although theories of aging postulate a non-arithmetic aging decline process, at least in biological aging, ${ }^{45,46}$ our analysis assumes that the decline maintains a constant rate from 55 through 102 years, as also assumed by Terry and Katzman, ${ }^{41}$ when they established synaptic density changes through normal aging. But we are aware of the weaknesses of our calculations, which must be considered as an attempt to disentangle age, pathology, and education in non-representative samples of "normal" individuals from 55 through 102 years (who were not assessed from neuropsychological perspectives), and clinical MCI and AD samples.

Nevertheless, it is important to emphasize the limit of these results, already mentioned in our 'Participants' section; 
since we are re-analyzing four studies (two population and two clinical studies), although measures and procedures are identical for assessing cognitive plasticity, our criteria for inclusion are not exactly the same (those "healthy" individuals and 90+ years and older). Perhaps this fact could justify the broad differences in cognitive plasticity between the $90+$ and 75-89 years groups.

In conclusion, plasticity or learning capacities is present across old age, even in nonagenarian elders, but this plasticity declines through the normal aging process. MCI individuals and those with AD also show plasticity in the sense that they can improve their memory performance through learning. Nonagenarian individuals show a similar learning curve to those of MCI patients. "Normal" individuals from 55 through 89 years significantly differ in their learning curves from both pathological groups. Those pathological groups significantly differ from "normal" groups in the interference phenomenon. This effect is dramatic in AD patients; a "de-learning"effect appeared only in those individuals as a pathognomonic sign.

\section{Acknowledgments}

This study has been granted by the Research General Direction, MICINN: Project SEJ-2006-14438/PSIC; IMSERSO I+D+I Projects: 15-05 and 35-06. Our gratitude to Professor James Juola (University of Kansas) for his comments on this manuscript.

\section{Disclosure}

The authors report no conflicts of interest in this work.

\section{References}

1. Baltes PB, Sowarka D, Kliegl R. Cognitive training research on fluid intelligence in old age: what can older adult achieve by themselves? Psychol Aging. 1989;4(2):217-221.

2. Baltes MM, Raykov T. Prospective validity of cognitive plasticity in the diagnosis of mental status: A structural equation model. Neuropsychology. 1996;10:549-556.

3. Salthouse TA. Speed mediation of adult age differences in cognition. Dev Psychol. 1996;29:722-738.

4. Schaie KW. What can we learn from longitudinal studies of adult development? Res Hum Dev. 2005;2(3):133-158.

5. Vygotsky LS. Mind in Society. The Development of Higher Psychological Processes. Cole M, John-Steiner S, Scriber S, Souberman E, editors and translators. Cambridge, MA: Harvard University Press; 1978.

6. Embretson SE. Measuring and validating cognitive modifiability as an ability. JEM. 1992;29(1):25-50.

7. Cuddy A, Norton MI, Fiske ST. This old stereotype: The pervasiveness and persistence of the elderly stereotype. $J$ Soc Issues. 2005;61(2): 267-283.

8. Doidge N. The Brain That Changes Itself. New York: Viking Press; 2009.

9. Fernández-Ballesteros R, Calero MD. The assessment of learning potential: the EPA instrument. In: Lidz C, Elliot J, editors. Dynamic Assessment: Prevailing Models and Applications. Greenwich, CT: JAI Press; 2000:293-323.
10. Mayr U. Introduction to the special section on cognitive plasticity in the aging mind. Psychol Aging. 2008;23(4):681-683.

11. Calero MD, Navarro E. Relationships between plasticity, mild cognitive impairment and cognitive decline. Arc Clin Neuropsychol. 2004;19(5): 653-660.

12. Fernández-Ballesteros R, Zamarrón MD, Calero MD, Tárraga L. Cognitive plasticity and cognitive impairment. In: FernándezBallesteros R, editor. GeroPsychology. European Perspectives for an Ageing World. Göttingen, Germany: Hogrefe and Huber; 2007: $145-164$.

13. Kliegl R, Smith J, Baltes PB. On the locus and process of magnification of age differences during mnemonic training. Dev Psychol. 1990;26: 894-904.

14. Singer T, Lindenberger U, Baltes PB. Plasticity of memory for new learning in very old age: A story of major loss? Psychol Aging. 2003; 18(2):306-317.

15. Baltes PB, Smith J. New frontiers in the future of aging: from successful aging of the young old to the dilemmas of the fourth age. Gerontology. 2003;49(2):123-135.

16. Yang L, Krampe RT, Baltes PB. Basic forms of cognitive plasticity extended into the oldest-old: retest learning, age and cognitive functioning. Psychol Aging. 2006;21(2):372-378.

17. Petersen R. Mild cognitive impairment: transition between aging and Alzheimer's disease. Neurología. 2000;15(3):93-101.

18. Boeve B, McCormick RN, Smith G, et al. Mild cognitive impairment in the oldest old. Neurology. 2003;60(3):477-480.

19. Baltes MM, Kühl KP, Sowarka D. Testing the limits of cognitive reserve capacity a promising strategy for early diagnosis of dementia? J Gerontol. 1992;47(3):165-167.

20. Lindenberger $U$, Reischies FM. Limits and potentials of intellectual functioning in old age. In: Baltes PB, Mayer KU, editors. The Berlin Aging Study. Aging from 70 to 100. Cambridge, UK: Cambridge University Press; 1999:329-360.

21. Fernández-Ballesteros R, Zamarrón MD, Tárraga L, Moya R, Iñiguez J. Learning Potential in Healthy, Mild Cognitive Impairment subjects and in Alzheimer patients. Eur Psychol. 2003;8:148-160.

22. Rey H. [Clinical Examination in Psychology.] Paris, France: Press Universitaire de France; 1964. French.

23. Fernández-Ballesteros R, Zamarrón MD, Tárraga L. Learning potential: a new method for assessing cognitive impairment. Int Psychogeriatr. 2005;17(1):119-128.

24. Zamarrón MD, Tárraga L, Fernández-Ballesteros R. Changes in reserve capacity in Alzheimer's disease patients attending psycho-stimulation training programs. Psychology in Spain. 2009;13:8-54.

25. Fernández-Ballesteros R, Calero MD. Training effects on intelligence of older persons. Arch Gerontol Geriatr. 1995;20(2):135-148.

26. Christensen $\mathrm{H}$, Korten $\mathrm{AE}$, Jorm AF, et al. Education and decline in cognitive performance: compensatory but not protective. Int J Geriatr Psychiatry. 1997;12(3):323-330.

27. Kliegel M, Zimpric D, Rott C. Life-long intellectual activities mediate the predictive effect of early education on cognitive impairment in centenarians: a retrospective study. Aging Ment Health. 2004;8(5): 430-437.

28. Ngandu T, von Strauss E, Helkala EL, et al. Education and dementia: what lies behind the association? Neurology. 2007;69(14):1442-1450.

29. Schmand B, Smith J, Lindenboom J, et al. Low education is a genuine risk factor for accelerated memory decline and dementia. $J$ Clin Epidemiol. 1997;50(9):1025-1033.

30. Fernandez-Ballesteros R, Zamarrón MD, López MD, et al. [Successful aging: criteria and predictors.] Psychology in Spain. 2011;15(1):94-101. Spanish.

31. Mahoney FI, Barthel DW. Functional evaluation: the Barthel Index. Md State Med J. 1965;14:64-65.

32. Pfeiffer A. A short portable mental status questionnaire for the assessment of organic brain deficit in elderly patients. J Am Geriatr Soc. 1975;23(10):433-441.

33. Díez-Nicolás J. Los Mayores en la Comunidad de Madrid. Madrid, Spain: Obra Social Caja Madrid; 1996. Spanish. 
34. Petersen R, Smith G, Waring SC, Ivnik R, Tangalos TG, Kokmen E. Mild cognitive impairment: clinical characterization and outcome. Arch Neurol. 1999;56(3):303-308.

35. American Psychiatric Association. Diagnostic and Statistical Manual of Mental Disorders IV. Washington, DC: American Psychiatric Association; 1994

36. McKhann G, Drachman D, Folstein M, Katzman R, Price D, Stadlan EM. Clinical diagnosis of Alzheimer's disease: report of the NINCDSADRDA Work Group under the auspices of Department of Health and Human Services Task Force on Alzheimer's Disease. Neurology. 1984;34(7):939-944.

37. Folstein MF, Folstein SE, McHugh PR. Minimental state. A practical method for grading the cognitive state of patients for the clinician. J Psychiatr Res. 1975;12(3):189-198.

38. Reisberg B, Ferris SH, de Leon MJ, Crook T. The Global Deterioration Scale for assessment of primary degenerative dementia. Am J Psychiatry. 1982;139(9):1136-1139.

39. Hachinski VC, Iliff LD, Zihlka E, et al. Cerebral blood flow in dementia. Arch Neurol. 1975;32(9):632-637.
40. Delis D, Kramer J, Kaplan E, Ober B. California Verbal Learning Test: Adult Version Manual. San Antonio, TX: The Psychological Corporation; 1987.

41. Terry RD, Katzman R. Life span and synapses: will there be a primary senile dementia? Neurobiol Aging. 2001;22(3):347-348.

42. Molina MA, Schettini R, Bravo MD, Zamarrón MD, Fernández-Ballesteros R. Cognitive activies and cognitive functioning in the elderly. Rev Esp Geriatr Gerontol. 2011. [Epub ahead of print.]

43. Schreiber M, Schneider R. Cognitive plasticity in people at risk for dementia: optimising the testing-the-limits-approach. Aging Men Health. 2007;11(1):75-81.

44. Tárraga L, Boada M, Modinos G, et al. A randomized pilot study to assess the efficacy of an interactive, multimedia tool of cognitive stimulation in Alzheimer's Disease. J Neurol Neurosurg Psychiatry. 2006;77(10):1116-1121.

45. Mayr E. This is Biology. The Science of the Living World. Cambridge, MA Harvard University Press; 1997.

46. Medina JJ. The Clock of Ages. Cambridge, UK: Cambridge University Press; 1997.
Clinical Interventions in Aging

\section{Publish your work in this journal}

Clinical Interventions in Aging is an international, peer-reviewed journal focusing on evidence-based reports on the value or lack thereof of treatments intended to prevent or delay the onset of maladaptive correlates of aging in human beings. This journal is indexed on PubMed Central, MedLine, the American Chemical Society's 'Chemical Abstracts

\section{Dovepress}

Service' (CAS), Scopus and the Elsevier Bibliographic databases. The manuscript management system is completely online and includes a very quick and fair peer-review system, which is all easy to use. Visit http://www.dovepress.com/testimonials.php to read real quotes from published authors. 